\title{
OFDM-IM-Based Spectrum Sharing for Cognitive Radio Networks
}

\author{
Qiang $\mathrm{Li}^{\dagger}$, Miaowen $\mathrm{Wen}^{\dagger}$, Shuping Dang ${ }^{\#}$, Ertugrul Basar ${ }^{\$}$, H. Vincent Poor ${ }^{\ddagger}$, and Fangjiong Chen ${ }^{\dagger}$ \\ ${ }^{\dagger}$ School of Electronic and Information Engineering, \\ South China University of Technology, Guangzhou 510640, China \\ \#Computer, Electrical and Mathematical Science and Engineering Division, \\ King Abdullah University of Science and Technology, Thuwal 23955-6900, Kingdom of Saudi Arabia \\ $\$$ CoreLab, Department of Electrical and Electronics Engineering, Koç University, Sariyer 34450, Istanbul, Turkey \\ $\ddagger$ Department of Electrical Engineering, Princeton University, Princeton, NJ 08544, USA \\ Email: eeqiangli@mail.scut.edu.cn, eemwwen@scut.edu.cn, shuping.dang@kaust.edu.sa, \\ ebasar@ku.edu.tr, poor@princeton.edu, eefjchen@scut.edu.cn
}

\begin{abstract}
In this paper, a novel spectrum sharing scheme, based on orthogonal frequency division multiplexing with index modulation (OFDM-IM), is proposed for cognitive radio networks. In the considered model, the primary transmitter communicates with the primary receiver with the aid of an amplify-and-forward (AF) relay by transmitting OFDM-IM signals. Meanwhile, the secondary transmitter passively senses the spectrum and transmits its own information over those inactive subcarriers of the primary network to the secondary receiver. Through the proposed protocol, inactive subcarriers inherent in the existing OFDM-IM systems can be fully exploited, thus improving the spectral efficiency. To evaluate the performance, closed-form upper bounds on the bit error rate (BER) are derived for both users utilizing the optimal maximum-likelihood detection. Simulation results corroborate the analysis and show that the proposed scheme has the potential of outperforming OFDM-IM-AF in terms of BER with higher spectral efficiency.

Keywords - Amplify-and-forward relaying, cognitive radio, index modulation, OFDM, spectrum sharing.
\end{abstract}

\section{INTRODUCTION}

Cognitive radio $(\mathrm{CR})$ has been proposed as a promising approach for improving spectral efficiency through spectrum sharing. In CR networks, unlicensed secondary users (SUs) are intelligent devices, which sense their surrounding electromagnetic environments and dynamically adjust certain radio operating parameters for the access to the spectrum owned by licensed primary users (PUs) [1].

OFDM-IM is a competitive frequency-domain IM transmission technique, which activates only a subset of all available subcarriers to convey $M$-ary modulated symbols and utilizes the resulting subcarrier activation patterns (SAPs) to transmit additional information bits [2]-[4]. It has been shown in the literature that OFDM-IM not only maintains the advantages of classical OFDM, but also achieves superiority in terms of BER and flexibility in system configuration. Recently, OFDM-IM has been incorporated with amplify-and-forward (AF)/decodeand-forward relaying technique to extend the coverage and improve system reliability [5], [6]. In view of two informationcarrying units, the potential of OFDM-IM is investigated in overlay OFDM-based CR networks, where the PU transmits classical OFDM signals, and the SU employs OFDM-IM to forward the PU's information via $M$-ary symbols and convey its own information through SAPs [7]. However, how to explore the opportunity to access the idle spectrum when the PU employs OFDM-IM for information transmission, is still an open topic. In particular, since partial subcarriers in OFDM-IM are inherently inactive (spectrum holes), they can potentially be used by SUs for improving the spectral efficiency.

To fill the aforementioned gap, motivated by the concept of $\mathrm{CR}$, we propose a novel spectrum sharing scheme in this paper by which spectrum holes in OFDM-IM systems can be utilized by a SU. Specifically, in the first phase, the primary transmitter (PT) broadcasts OFDM-IM signals to an AF relay and the secondary transmitter (ST). Upon receiving this signal, the ST performs spectrum sensing. In the second phase, the relay amplifies and forwards the received signal to the primary receiver (PR). Meanwhile, the ST transmits its own information on those inactive subcarriers of the primary network to the secondary receiver (SR). Through the proposed protocol, inactive subcarriers inherent in the existing OFDMIM systems can be fully exploited, thus improving the spectral efficiency.

The remainder of this paper is organized as follows. The system model of an OFDM-IM-based CR network is given in Section II, followed by the performance analysis in Section III. Section IV presents computer simulation results and finally Section V concludes the paper.

\section{SySTEM MODEL}

Fig. 1 depicts the system model of OFDM-IM-based CR network, in which the ST is close to the PT, and all nodes operate in the half-duplex mode with a single transmit/receive

\footnotetext{
${ }^{1}$ Notation: Column vectors are denoted by lowercase bold letters. Superscript ${ }^{T}$ stands for transpose. $\mathcal{C N}\left(0, \sigma^{2}\right)$ represents the complex Gaussian distribution with zero mean and variance $\sigma^{2}$. The probability of an event and the probability density function (PDF) of a random variable are denoted by $\operatorname{Pr}(\cdot)$ and $f(\cdot)$, respectively. $E\{\cdot\}$ denotes expectation. $\lfloor\cdot\rfloor$ and $C(\cdot, \cdot)$ are the floor function and the binomial coefficient, respectively. $Q(\cdot)$ and $\operatorname{Ei}(\cdot)$ represent the Gaussian $Q$-function and the exponential integral function, respectively.
} 


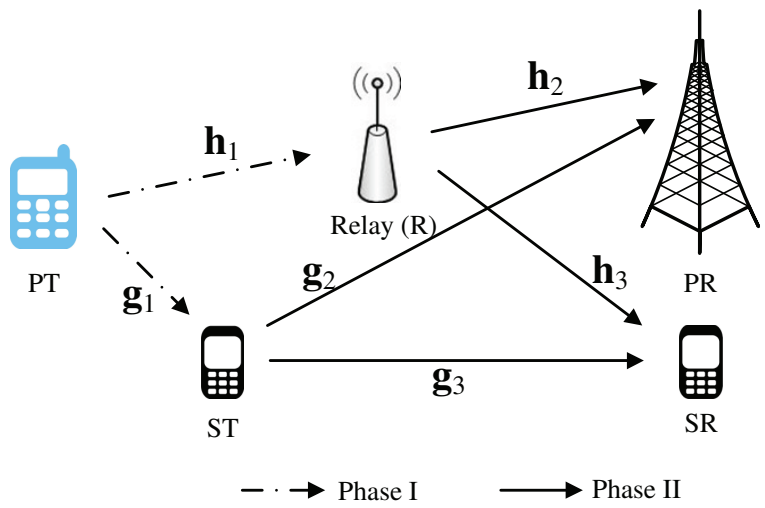

Fig. 1. System model of OFDM-IM-based CR network.

antenna. Due to the broadband signal and rich scattering in the propagation environment, all channel links are assumed to experience frequency-selective Rayleigh fading, except the $\mathrm{PT} \rightarrow \mathrm{ST}$ link, which is modeled by frequency-selective Rician fading as the ST and PT are located close together.

The communication protocol consists of two phases. In the first phase, the PT transmits its information via OFDMIM signal to the relay (R) and the ST. According to [2], the formation of the OFDM-IM signal is the same as that of OFDM except that IM is included in the generation of frequency-domain OFDM block. Specifically, for an OFDMIM system with $N$ subcarriers, the $N \times 1$ main OFDM block is created by stacking $G$ subblocks, each of which is formed by performing IM within $n=N / G$ subcarriers. Since the processes in all subblocks are the same and independent of each other, we focus only on a single subblock, say, the $\beta$ th one, where $\beta \in\{1, \ldots, G\}$. The information bits to be transmitted in the $\beta$ th subblock are divided into two parts for further processing. The first part, consisting of $p_{1}=$ $\left\lfloor\log _{2}(C(n, k))\right\rfloor$ bits, is used to determine the positions of $k$ active subcarriers within the $\beta$ th subblock, forming the active indices (i.e., the SAP)

$$
\mathcal{I}^{(\beta)}=\left\{i^{(\beta)}(1), \ldots, i^{(\beta)}(k)\right\} .
$$

The mapping between $\left\lfloor\log _{2}(C(n, k))\right\rfloor$ bits and $\mathcal{I}^{(\beta)}$ can be easily realized by either a look-up table or the combinatorial method [2]. The second part with $p_{2}=k \log _{2}(M)$ bits is mapped to the data symbol vector

$$
\mathbf{s}_{p}^{(\beta)}=\left[s_{p}^{(\beta)}(1), \ldots, s_{p}^{(\beta)}(k)\right]^{T},
$$

according to a normalized $M$-ary phase shift keying ( $\mathrm{P}$ $\mathrm{SK})$ /quadrature amplitude modulation signal constellation $\mathcal{X}$, where $s_{p}^{(\beta)}(\lambda) \in \mathcal{X}$ is to be transmitted over the subcarrier with index $i^{(\beta)}(\lambda), \lambda=1, \ldots, k$. After obtaining $\mathcal{I}^{(\beta)}$ and $\mathbf{s}^{(\beta)}$ for all $\beta$, we have the $N \times 1$ main OFDM block for the PT as

$$
\begin{aligned}
\mathbf{x}_{p t} & =\left[X_{p}(1), \ldots, X_{p}(N)\right]^{T} \\
& =\left[\left(\mathbf{x}_{p}^{(1)}\right)^{T}, \ldots,\left(\mathbf{x}_{p}^{(G)}\right)^{T}\right]^{T},
\end{aligned}
$$

where $\mathbf{x}_{p}^{(\beta)}=\left[X_{p}^{(\beta)}(1), \ldots, X_{p}^{(\beta)}(n)\right]^{T}$ is the $\beta$ th $n \times 1$ subblock with $X_{p}^{(\beta)}(\alpha) \in\{0, \mathcal{X}\}, \alpha=1, \ldots, n$.

The received signal at $\mathrm{R}$ for the $\beta$ th subblock in the frequency domain can be expressed as

$$
y_{r}^{\beta}(\alpha)=h_{1}^{(\beta)}(\alpha) X_{p}^{(\beta)}(\alpha)+n_{r}^{(\beta)}(\alpha), \quad \alpha=1, \ldots, n
$$

where $h_{1}^{(\beta)}(\alpha)$ and $n_{r}^{(\beta)}(\alpha)$ are the channel frequency response (CFR) of the PT $\rightarrow \mathrm{R}$ link and the additive white Gaussian noise (AWGN) sample at $\mathrm{R}$ on the $\alpha$ th subcarrier of the $\beta$ th subblock, respectively. It is assumed that $h_{1}^{(\beta)}(\alpha)$ and $n_{r}^{(\beta)}(\alpha)$ follow the distributions $\mathcal{C N}\left(0, \sigma_{1}^{2}\right)$ and $\mathcal{C N}\left(0, N_{0}\right)$, respectively. We define the transmit signal-to-noise ratio (SNR) per subcarrier as $\rho=k /\left(n N_{0}\right)$.

In the second phase, $\mathrm{R}$ relays $y_{r}^{(\beta)}(\alpha), \beta=1, \ldots, G, \alpha=$ $1, \ldots, n$, to the PR with an amplification factor $A$. With the assumption that $\mathrm{R}$ has the same transmit power as the PT and the fixed-gain AF relaying is adopted [8], $A$ can be written as

$$
A=\sqrt{\frac{k}{k \sigma_{1}^{2}+n N_{0}}} .
$$

Meanwhile, after perfectly sensing the positions of inactive subcarriers of the PU, the ST modulates its own information of $p_{3}=(n-k) \log _{2}(M)$ bits into the symbol vector

$$
\mathbf{s}_{s}^{(\beta)}=\left[s_{s}^{(\beta)}(1), \ldots, s_{s}^{(\beta)}(n-k)\right]^{T},
$$

and transmits it over the subcarriers of indices $\overline{\mathcal{I}}^{(\beta)}$, yielding the transmit block

$$
\begin{aligned}
\mathbf{x}_{s t} & =\left[X_{s}(1), \ldots, X_{s}(N)\right]^{T} \\
& =\left[\left(\mathbf{x}_{s}^{(1)}\right)^{T}, \ldots,\left(\mathbf{x}_{s}^{(G)}\right)^{T}\right]^{T},
\end{aligned}
$$

where $\mathbf{x}_{s}^{(\beta)}=\left[X_{s}^{(\beta)}(1), \ldots, X_{s}^{(\beta)}(n)\right]^{T}$ is the $\beta$ th $n \times 1$ subblock with $X_{s}^{(\beta)}(\alpha) \in\{0, \mathcal{X}\}, \alpha=1, \ldots, n$. Note that the indices of non-zero elements in $\mathbf{x}_{s t}$ are complementary to those in $\mathbf{x}_{p t}$. The frequency-domain received signal at the PR for the $\beta$ th subblock is given by

$$
\begin{aligned}
y_{p r}^{(\beta)}(\alpha)= & A h_{2}^{(\beta)}(\alpha) y_{r}^{(\beta)}(\alpha)+g_{2}^{(\beta)}(\alpha) X_{s}^{(\beta)}(\alpha)+n_{p r}^{(\beta)}(\alpha) \\
= & A h_{1}^{(\beta)}(\alpha) h_{2}^{(\beta)}(\alpha) X_{p}^{(\beta)}(\alpha)+g_{2}^{(\beta)}(\alpha) X_{s}^{(\beta)}(\alpha) \\
& +A h_{2}^{(\beta)}(\alpha) n_{r}^{(\beta)}(\alpha)+n_{p r}^{(\beta)}(\alpha), \quad \alpha=1, \ldots, n
\end{aligned}
$$

where $h_{2}^{(\beta)}(\alpha)$ and $g_{2}^{(\beta)}(\alpha)$ are the CFRs of the $\mathrm{R} \rightarrow \mathrm{PR}$ and $\mathrm{ST} \rightarrow \mathrm{PR}$ links on the $\alpha$ th subcarrier of the $\beta$ th subblock, following the distributions $\mathcal{C N}\left(0, \sigma_{2}^{2}\right)$ and $\mathcal{C N}\left(0, \phi_{2}^{2}\right)$, respectively; $n_{p r}^{(\beta)}(\alpha)$ is the AWGN sample at the PR on the $\alpha$ th subcarrier of the $\beta$ th subblock with the distribution $\mathcal{C N}\left(0, N_{0}\right)$. After noise normalization, (8) can be rewritten as

$$
\begin{aligned}
\bar{y}_{p r}^{(\beta)}(\alpha)= & \frac{A h_{1}^{(\beta)}(\alpha) h_{2}^{(\beta)}(\alpha)}{\sqrt{1+A^{2}\left|h_{2}^{(\beta)}(\alpha)\right|^{2}}} X_{p}^{(\beta)}(\alpha) \\
& +\frac{g_{2}^{(\beta)}(\alpha)}{\sqrt{1+A^{2}\left|h_{2}^{(\beta)}(\alpha)\right|^{2}}} X_{s}^{(\beta)}(\alpha)+n^{(\beta)}(\alpha),
\end{aligned}
$$


where $n^{(\beta)}(\alpha)$ is a complex-valued Gaussian variable with zero mean and variance $N_{0}$. Due to the encoding independence among different subblocks, the detection for all subblocks can be performed in parallel. For brevity, the superscript $(\beta)$ is omitted in the sequel. From (9), the optimal maximumlikelihood (ML) detection for the PU at the PR can be derived as

$$
\begin{aligned}
\left(\hat{\mathcal{I}}, \hat{\mathbf{s}}_{p}, \hat{\mathbf{s}}_{s}\right)=\underset{\mathcal{I}, \mathbf{s}_{p}, \mathbf{s}_{s}}{\arg \min } \sum_{\alpha=1}^{n} \mid \bar{y}_{p r}(\alpha) & \\
& -\frac{A h_{1}(\alpha) h_{2}(\alpha)}{\sqrt{1+A^{2}\left|h_{2}(\alpha)\right|^{2}}} X_{p}(\alpha) \\
& -\left.\frac{g_{2}(\alpha)}{\sqrt{1+A^{2}\left|h_{2}(\alpha)\right|^{2}}} X_{s}(\alpha)\right|^{2} .
\end{aligned}
$$

Similar to (9) and (10), the received signal and ML detection for the SU at the SR can be easily formulated correspondingly, which are omitted here to avoid redundancy.

Remark: The ML detector [2] and the energy detector [9] can be used for spectrum sensing (SAP detection) at the ST. Though not shown in this paper, practical spectrum sensing methods, such as the ML and energy detectors, are able to approach and even achieve the optimal overall performance that is obtained with perfect spectrum sensing over Rician fading channels. For this reason and for ease of performance analysis for perfect spectrum sensing, we assume perfect spectrum sensing instead of practical spectrum sensing at the ST in this paper.

\section{PERformance AnAlysis}

In this section, we derive closed-form upper bounds on the BER of the PU and SU utilizing ML detection at the PR. The analysis at the SR can be implemented in a similar manner.

Let us first consider the joint conditional pairwise error probability (PEP), i.e., $\operatorname{Pr}\left(\mathbf{x}_{p} \rightarrow \hat{\mathbf{x}}_{p}, \mathbf{x}_{s} \rightarrow \hat{\mathbf{x}}_{s} \mid \mathbf{h}_{1}, \mathbf{h}_{2}, \mathbf{g}_{2}\right)$, which represents the probability for the event that the transmitted $\mathbf{x}_{p}$ and $\mathbf{x}_{s}$ are erroneously detected as $\hat{\mathbf{x}}_{p}$ and $\hat{\mathbf{x}}_{s}$, respectively. From (10), we have

$$
\begin{aligned}
& \operatorname{Pr}\left(\mathbf{x}_{p} \rightarrow \hat{\mathbf{x}}_{p}, \mathbf{x}_{s} \rightarrow \hat{\mathbf{x}}_{s} \mid \mathbf{h}_{1}, \mathbf{h}_{2}, \mathbf{g}_{2}\right) \\
& =\operatorname{Pr}\left(\sum_{\alpha=1}^{n} \mid \bar{y}_{p r}(\alpha)-\frac{A h_{1}(\alpha) h_{2}(\alpha)}{\sqrt{1+A^{2}\left|h_{2}(\alpha)\right|^{2}}} X_{p}(\alpha)\right. \\
& -\left.\frac{g_{2}(\alpha)}{\sqrt{1+A^{2}\left|h_{2}(\alpha)\right|^{2}}} X_{s}(\alpha)\right|^{2} \\
& >\sum_{\alpha=1}^{n} \mid \bar{y}_{p r}(\alpha)-\frac{A h_{1}(\alpha) h_{2}(\alpha)}{\sqrt{1+A^{2}\left|h_{2}(\alpha)\right|^{2}}} \hat{X}_{p}(\alpha) \\
& \left.-\left.\frac{g_{2}(\alpha)}{\sqrt{1+A^{2}\left|h_{2}(\alpha)\right|^{2}}} \hat{X}_{s}(\alpha)\right|^{2}\right) \\
& =Q\left(\sqrt{\frac{1}{2 N_{0}} \sum_{\alpha=1}^{n} T(\alpha)}\right) \text {, }
\end{aligned}
$$

where

$$
T(\alpha)=\frac{\left|A h_{1}(\alpha) h_{2}(\alpha) \Delta X_{p}(\alpha)+g_{2}(\alpha) \Delta X_{s}(\alpha)\right|^{2}}{1+A^{2}\left|h_{2}(\alpha)\right|^{2}}
$$

with $\Delta X_{p}(\alpha)=X_{p}(\alpha)-\hat{X}_{p}(\alpha)$ and $\Delta X_{s}(\alpha)=X_{s}(\alpha)-$ $\hat{X}_{s}(\alpha)$. According to the following approximation of the $Q$ function: $Q(x) \approx 1 / 12 \cdot \exp \left(-x^{2} / 2\right)+1 / 4 \cdot \exp \left(-2 x^{2} / 3\right)$, (11) can be simplified as

$$
\begin{aligned}
& \operatorname{Pr}\left(\mathbf{x}_{p} \rightarrow \hat{\mathbf{x}}_{p}, \mathbf{x}_{s} \rightarrow \hat{\mathbf{x}}_{s} \mid \mathbf{h}_{1}, \mathbf{h}_{2}, \mathbf{g}_{2}\right) \\
& \approx \frac{1}{12} \prod_{\alpha=1}^{n} \exp \left(-q_{1} T(\alpha)\right)+\frac{1}{4} \prod_{\alpha=1}^{n} \exp \left(-q_{2} T(\alpha)\right),
\end{aligned}
$$

where $q_{1}=1 /\left(4 N_{0}\right)$ and $q_{2}=1 /\left(3 N_{0}\right)$. Thus, the unconditional PEP can be approximated as

$$
\begin{aligned}
& \operatorname{Pr}\left(\mathbf{x}_{p} \rightarrow \hat{\mathbf{x}}_{p}, \mathbf{x}_{s} \rightarrow \hat{\mathbf{x}}_{s}\right) \\
& \approx \frac{1}{12} \prod_{\alpha=1}^{n} E_{h_{1}, h_{2}, g_{2}}\left\{\exp \left(-q_{1} T(\alpha)\right)\right\} \\
& \quad+\frac{1}{4} \prod_{\alpha=1}^{n} E_{h_{1}, h_{2}, g_{2}}\left\{\exp \left(-q_{2} T(\alpha)\right)\right\} .
\end{aligned}
$$

For the calculation of $E_{h_{1}, h_{2}, g_{2}}\left\{\exp \left(-q_{i} T(\alpha)\right)\right\}, i=1,2$, we can figure out four scenarios, as described below.

- When $\Delta X_{p}(\alpha)=0$ and $\Delta X_{s}(\alpha)=0$, we have $T(\alpha)=0$ and $E_{h_{1}, h_{2}, g_{2}}\left\{\exp \left(-q_{i} T(\alpha)\right)\right\}=1$.

- When $\Delta X_{p}(\alpha) \neq 0$ and $\Delta X_{s}(\alpha) \neq 0$, since $h_{1}(\alpha)$, $h_{2}(\alpha)$, and $g_{2}(\alpha)$ are independent of each other, we have

$$
\begin{aligned}
& E_{h_{1}, h_{2}, g_{2}}\left\{\exp \left(-q_{i} T(\alpha)\right)\right\} \\
& =E_{h_{2}}\left\{E_{h_{1} \mid h_{2}}\left\{E_{g_{2} \mid h_{1}, h_{2}}\left\{\exp \left(-q_{i} T(\alpha)\right)\right\}\right\}\right\} .
\end{aligned}
$$

Given $h_{1}(\alpha)$ and $h_{2}(\alpha)$, we observe that $\left|A h_{1}(\alpha) h_{2}(\alpha) \Delta X_{p}(\alpha)+g_{2}(\alpha) \Delta X_{s}(\alpha)\right|^{2} \quad$ is a noncentral chi-square random variable, whose moment generating function (MGF) is given by [10]

$$
\begin{aligned}
\mathcal{M}(\omega)= & \frac{1}{1-\omega \phi_{2}^{2}\left|\Delta X_{s}(\alpha)\right|^{2}} \\
& \times \exp \left(\frac{\omega\left|A h_{1}(\alpha) h_{2}(\alpha) \Delta X_{p}(\alpha)\right|^{2}}{1-\omega \phi_{2}^{2}\left|\Delta X_{s}(\alpha)\right|^{2}}\right) .
\end{aligned}
$$

Hence, we obtain

$$
\begin{aligned}
& E_{g_{2} \mid h_{1}, h_{2}}\left\{\exp \left(-q_{i} T(\alpha)\right)\right\} \\
& =\frac{1+A^{2}\left|h_{2}(\alpha)\right|^{2}}{1+A^{2}\left|h_{2}(\alpha)\right|^{2}+q_{i} \phi_{2}^{2}\left|\Delta X_{s}(\alpha)\right|^{2}} \\
& \quad \times \exp \left(-\frac{q_{i} A^{2}\left|h_{2}(\alpha)\right|^{2}\left|\Delta X_{p}(\alpha)\right|^{2}\left|h_{1}(\alpha)\right|^{2}}{1+A^{2}\left|h_{2}(\alpha)\right|^{2}+q_{i} \phi_{2}^{2}\left|\Delta X_{s}(\alpha)\right|^{2}}\right) .
\end{aligned}
$$

Since the MGF of $\left|h_{1}(\alpha)\right|^{2}$ is

$$
\mathcal{M}(\omega)=\frac{1}{1-\omega \sigma_{1}^{2}}
$$

using the MGF approach again yields (19), which is shown at the top of the next page. Finally, by averaging 


$$
E_{h_{1} \mid h_{2}}\left\{E_{g_{2} \mid h_{1}, h_{2}}\left\{\exp \left(-q_{i} T(\alpha)\right)\right\}\right\}=\frac{1+A^{2}\left|h_{2}(\alpha)\right|^{2}}{1+q_{i} \phi_{2}^{2}\left|\Delta X_{s}(\alpha)\right|^{2}+\left(A^{2}+q_{i} A^{2} \sigma_{1}^{2}\left|\Delta X_{p}(\alpha)\right|^{2}\right)\left|h_{2}(\alpha)\right|^{2}}
$$

(19) over $\left|h_{2}(\alpha)\right|^{2}$, whose PDF is given by

$$
f(x)=\frac{1}{\sigma_{2}^{2}} \exp \left(-\frac{x}{\sigma_{2}^{2}}\right),
$$

we are led to [11, Eqs. (3.352-4, 3.353-5)]

$$
\begin{aligned}
& E_{h_{1}, h_{2}, g_{2}}\left\{\exp \left(-q_{i} T(\alpha)\right)\right\} \\
& =\frac{\exp \left(\gamma / \sigma_{2}^{2}\right) \operatorname{Ei}\left(-\gamma / \sigma_{2}^{2}\right)\left(A^{2} \gamma-1\right)+A^{2} \sigma_{2}^{2}}{A^{2} \sigma_{2}^{2}\left(1+q_{i} \sigma_{1}^{2}\left|\Delta X_{p}(\alpha)\right|^{2}\right)},
\end{aligned}
$$

where

$$
\gamma=\frac{1+q_{i} \phi_{2}^{2}\left|\Delta X_{s}(\alpha)\right|^{2}}{A^{2}\left(1+q_{i} \sigma_{1}^{2}\left|\Delta X_{p}(\alpha)\right|^{2}\right)} .
$$

- When $\Delta X_{p}(\alpha)=0$ and $\Delta X_{s}(\alpha) \neq 0, T(\alpha)$ becomes

$$
T(\alpha)=\frac{\left|g_{2}(\alpha)\right|^{2}\left|\Delta X_{s}(\alpha)\right|^{2}}{1+A^{2}\left|h_{2}(\alpha)\right|^{2}}
$$

Considering that $\left|g_{2}(\alpha)\right|^{2}$ and $\left|h_{2}(\alpha)\right|^{2}$ are independent exponential random variables with means equal to $\phi_{2}^{2}$ and $\sigma_{2}^{2}$, respectively, we arrive at

$$
\begin{aligned}
& E_{h_{1}, h_{2}, g_{2}}\left\{\exp \left(-q_{i} T(\alpha)\right)\right\} \\
& =E_{h_{2}}\left\{E_{g_{2} \mid h_{2}}\left\{\exp \left(-q_{i} T(\alpha)\right)\right\}\right\} \\
& =E_{h_{2}}\left\{\frac{1+A^{2}\left|h_{2}(\alpha)\right|^{2}}{1+A^{2}\left|h_{2}(\alpha)\right|^{2}+q_{i} \phi_{2}^{2}\left|\Delta X_{s}(\alpha)\right|^{2}}\right\} \\
& =\frac{1}{\sigma_{2}^{2}} \exp \left(\gamma / \sigma_{2}^{2}\right) \operatorname{Ei}\left(-\gamma / \sigma_{2}^{2}\right)\left(\gamma-1 / A^{2}\right)+1,
\end{aligned}
$$

where

$$
\gamma=\frac{1+q_{i} \phi_{2}^{2}\left|\Delta X_{s}(\alpha)\right|^{2}}{A^{2}}
$$

- When $\Delta X_{p}(\alpha) \neq 0$ and $\Delta X_{s}(\alpha)=0, T(\alpha)$ becomes

$$
T(\alpha)=\frac{A^{2}\left|h_{1}(\alpha)\right|^{2}\left|h_{2}(\alpha)\right|^{2}\left|\Delta X_{p}(\alpha)\right|^{2}}{1+A^{2}\left|h_{2}(\alpha)\right|^{2}} .
$$

After some mathematical operations, we have

$$
\begin{aligned}
& E_{h_{1}, h_{2}, g_{2}}\left\{\exp \left(-q_{i} T(\alpha)\right)\right\} \\
& =E_{h_{2}}\left\{E_{h_{1} \mid h_{2}}\left\{\exp \left(-q_{i} T(\alpha)\right)\right\}\right\} \\
& =\frac{\exp \left(\gamma / \sigma_{2}^{2}\right) \operatorname{Ei}\left(-\gamma / \sigma_{2}^{2}\right)\left(A^{2} \gamma-1\right)+A^{2} \sigma_{2}^{2}}{A^{2} \sigma_{2}^{2}\left(1+q_{i} \sigma_{1}^{2}\left|\Delta X_{p}(\alpha)\right|^{2}\right)},
\end{aligned}
$$

where

$$
\gamma=\frac{1}{A^{2}\left(1+q_{i} \sigma_{1}^{2}\left|\Delta X_{p}(\alpha)\right|^{2}\right)}
$$

To sum up, $E_{h_{1}, h_{2}, g_{2}}\left\{\exp \left(-q_{i} T(\alpha)\right)\right\}$ can be expressed as (29), which is shown at the top of the next page, where $\gamma$ is given by (22). By substituting (29) into (14), the closed-form unconditional PEP can be derived. Finally, according to the union bounding technique, upper bounds on the BER of the $\mathrm{PU}$ and $\mathrm{SU}$ at the PR can be given by

$$
\begin{aligned}
P_{p u} \leq & \frac{1}{\left(p_{1}+p_{2}\right) \cdot 2^{p_{1}+p_{2}+p_{3}}} \\
& \times \sum_{\mathbf{x}_{p}, \mathbf{x}_{s}} \sum_{\hat{\mathbf{x}}_{p}, \hat{\mathbf{x}}_{s}} \operatorname{Pr}\left(\mathbf{x}_{p} \rightarrow \hat{\mathbf{x}}_{p}, \mathbf{x}_{s} \rightarrow \hat{\mathbf{x}}_{s}\right) N\left(\mathbf{x}_{p}, \hat{\mathbf{x}}_{p}\right),
\end{aligned}
$$

and

$$
\begin{aligned}
P_{s u} \leq & \frac{1}{p_{3} \cdot 2^{p_{1}+p_{2}+p_{3}}} \\
& \times \sum_{\mathbf{x}_{p}, \mathbf{x}_{s}} \sum_{\hat{\mathbf{x}}_{p}, \hat{\mathbf{x}}_{s}} \operatorname{Pr}\left(\mathbf{x}_{p} \rightarrow \hat{\mathbf{x}}_{p}, \mathbf{x}_{s} \rightarrow \hat{\mathbf{x}}_{s}\right) N\left(\mathbf{x}_{s}, \hat{\mathbf{x}}_{s}\right),
\end{aligned}
$$

respectively, where $N\left(\mathbf{x}_{p}, \hat{\mathbf{x}}_{p}\right)\left(N\left(\mathbf{x}_{s}, \hat{\mathbf{x}}_{s}\right)\right)$ denotes the number of erroneous bits when $\mathbf{x}_{p}\left(\mathbf{x}_{s}\right)$ is detected as $\hat{\mathbf{x}}_{p}\left(\hat{\mathbf{x}}_{s}\right)$.

\section{Simulation Results}

In this section, we perform Monte Carlo simulations to evaluate the uncoded BER performance of the PU and SU at the PR for the proposed OFDM-IM-based CR network. In all simulations, $\sigma_{1}^{2}=\sigma_{2}^{2}=\phi_{2}^{2}=1$, and all channel state information is assumed to be perfectly known at the receiver side. ML detection and binary PSK are employed for all schemes. For simplicity, we will refer to " $(n, k)$ " as the proposed scheme in which the PT activates $k$ out of $n$ subcarriers.

In Fig. 2, we present the BER curves of the PU and SU for $(n, k)=(4,1)$, where the PU and SU achieve the same data rate. The scheme of OFDM-IM-AF in the absence of the $\mathrm{SU}$ is also evaluated as a benchmark. To verify the analysis given in Section III, the BER upper bounds (30) and (31) are also depicted in the figure for the PU and SU, respectively. As seen from Fig. 2, both upper bounds agree with their computer simulation counterparts very well in the high SNR region. Moreover, we observe from Fig. 2 that the SU performs better than the PU for all SNR values. This is because the SU does not use SAPs that may be detected incorrectly to transmit information bits. Further, since the involvement of the SU enhances the reliability of SAP detection, it can been seen from Fig. 2 that the PU outperforms OFDM-IM-AF in the low-to-medium SNR region and they almost obtain the same asymptotic performance.

Fig. 3 gives the comparison results of the PU and SU with $n=4$ for different values of $k$. For the clarity of presentation, 


$$
E_{h_{1}, h_{2}, g_{2}}\left\{\exp \left(-q_{i} T(\alpha)\right)\right\}= \begin{cases}1, & \text { for }\left(\Delta X_{p}(\alpha), \Delta X_{s}(\alpha)\right)=(0,0) \\ \frac{\exp \left(\gamma / \sigma_{2}^{2}\right) \operatorname{Ei}\left(-\gamma / \sigma_{2}^{2}\right)\left(A^{2} \gamma-1\right)+A^{2} \sigma_{2}^{2}}{A^{2} \sigma_{2}^{2}\left(1+q_{i} \sigma_{1}^{2}\left|\Delta X_{p}(\alpha)\right|^{2}\right)}, & \text { for }\left(\Delta X_{p}(\alpha), \Delta X_{s}(\alpha)\right) \neq(0,0) .\end{cases}
$$

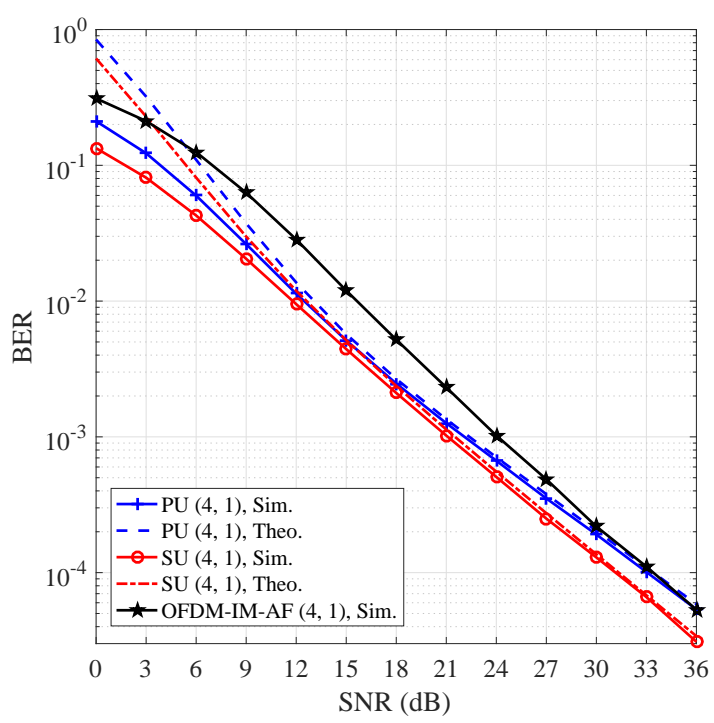

Fig. 2. BER performance of the PU and SU with $(n, k)=(4,1)$.

BER upper bounds are not given in Fig. 3. Similar to the observations in Fig. 2, for $k=1 / 2 / 3$, the PU performs worse than the SU throughout the considered SNR region. Furthermore, increasing $k$ results in worse BER performance for both the PU and SU.

\section{CONCLUSION}

In this paper, we have proposed OFDM-IM-based CR for spectrum sharing, in which the PU adopts AF aided OFDMIM for transmission, while the SU modulates its information on those inactive subcarriers of the PU. Spectrum utilization has been improved significantly without incurring mutual interference. Moreover, the performance of the PU has been enhanced with the existence of the SU. Tight upper bounds on the BER of the PU and SU have been derived in closed form. Computer simulations have verified the analysis and the effectiveness of the proposed scheme.

\section{ACKNOWLEDGMENT}

This work was supported in part by the National Natural Science Foundation of China under Grant 61871190, Grant 61671211, and Grant U1701265, in part by the Natural Science Foundation of Guangdong Province under Grant 2018B030306005, Grant 2016A030311024, and Grant 2016A030308006, in part by the Pearl River Nova Program of Guangzhou under Grant 201806010171, in part by the U.S. National Science Foundation under Grants CCF-0939370 and CCF-1513915, and in part by the China Scholarship Council under Grant 201806150074.

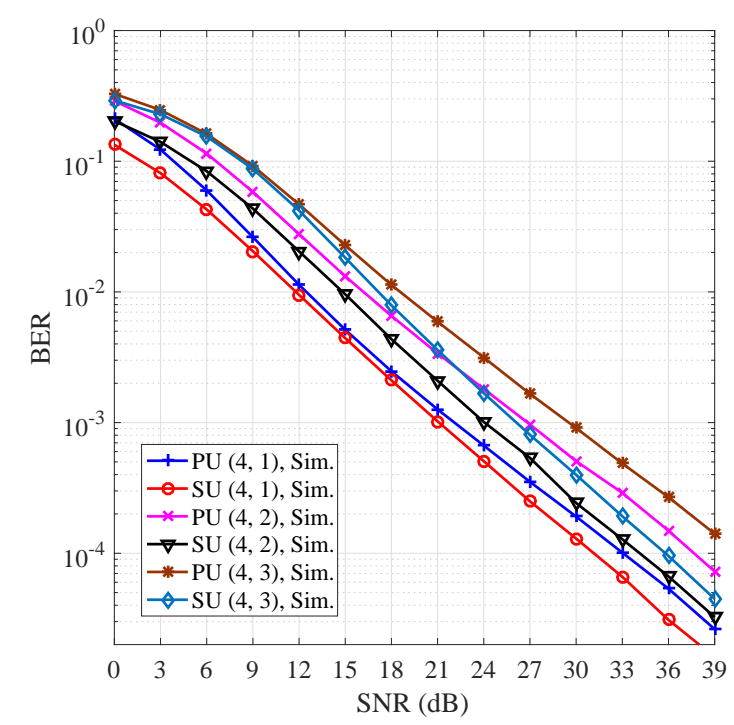

Fig. 3. BER performance of the PU and SU with $n=4$ and $k=1 / 2 / 3$.

\section{REFERENCES}

[1] S. Haykin, "Cognitive radio: Brain-empowered wireless communications," IEEE J. Select. Areas Commun., vol. 23, no. 2, pp. 201-220, Feb. 2005.

[2] E. Basar, U. Aygolu, E. Panayirci, and H. V. Poor, "Orthogonal frequency division multiplexing with index modulation," IEEE Trans. Signal Process., vol. 61, no. 22, pp. 5536-5549, Nov. 2013.

[3] M. Wen, X. Cheng, and L. Yang, Index Modulation for 5G Wireless Communications. Berlin, Germany: Springer, 2017.

[4] E. Basar, M. Wen, R. Mesleh, M. Di Renzo, Y. Xiao, and H. Haas, "Index modulation techniques for next-generation wireless networks," IEEE Access, vol. 5, pp. 16693-16746, Sep. 2017.

[5] S. Dang, J. P. Coon and G. Chen, "Adaptive OFDM with index modulation for two-hop relay-assisted networks," IEEE Trans. Wireless Commun., vol. 17, no. 3, pp. 1923-1936, Mar. 2018.

[6] S. Dang, J. Li, M. Wen, S. Mumtaz, and Z. Zhang, "OFDM-IM based dual-hop system using fixed-gain amplify-and-forward relay with preprocessing capability," IEEE Trans. Wireless Commun., vol. 18, no. 4, pp. 2259-2270, Apr. 2019.

[7] Q. Ma, P. Yang, L. Dan, X. He, Y. Xiao, and S. Li, "OFDM-IMaided cooperative relaying protocol for cognitive radio networks," in Proc. IEEE 18th Int. Workshop Signal Process. Adv. Wireless Commun. (SPAWC), Sapporo, Japan, Jul. 2017, pp. 1-5.

[8] M. O. Hasna and M.-S. Alouini, "A performance study of dual-hop transmissions with fixed gain relays," IEEE Trans. Wireless Commun., vol. 3, no. 6, pp. 1963-1968, Nov. 2004.

[9] T. V. Luong and Y. Ko, "Impact of CSI uncertainty on MCIK-OFDM: Tight closed-form symbol error probability analysis," IEEE Trans. Veh. Technol., vol. 67, no. 2, pp. 1272-1279, Feb. 2018.

[10] M. K. Simon, Probability Distributions Involving Gaussian Random Variables: A Handbook for Engineers and Scientists. Berlin, Germany: Springer, 2006.

[11] I. S. Gradshteyn and I. M. Ryzhik, Table of Integrals, Series, and Products, 7th ed., New York, NY, USA: Academic Press, 2007. 